\title{
Recurrent Gastric Adenocarcinoma
}

National Cancer Institute

\section{Source}

National Cancer Institute. Recurrent Gastric Adenocarcinoma. NCI Thesaurus. Code C154622.

The reemergence of gastric adenocarcinoma after a period of remission. 